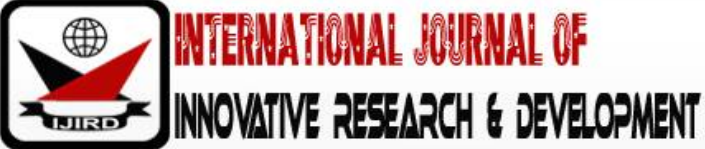

ISSN 2278 - 0211 (Online)

\section{Analysis of Diethylene Glycol and Decylamine as Demulsifiers for Treatment of Water-In-Oil Emulsions: Case Study of the Niger Delta, Nigeria}

\begin{tabular}{|c|}
\hline Chiamaka M. Chukwuemeka \\
Student, Department of Petroleum Engineering, \\
Federal University of Technology, Nigeria \\
Okechukwu C. David \\
Student, Department of Petroleum Engineering, \\
Federal University of Technology, Nigeria \\
Augustine C. Onele \\
Student, Department of Petroleum Engineering, \\
Federal University of Technology, Nigeria \\
Anthony O. Chikwe \\
Lecturer, Department of Petroleum Engineering, \\
Federal University of Technology, Nigeria
\end{tabular}

\begin{abstract}
:
Crude oil is made up of different hydrocarbon compounds. At different depths in the same reservoir, the properties of the crude oil differ from one another. During production of crude oil, emulsion is usually formed due interaction of water with emulsifying agents such as wax, as phaltenes, resins etc. present in crude oil. Water in oil emulsion (w/ o) poses serious problems during production and pipeline transportation of crude oil. To separate water from crude oil, demulsifier is required. This research work seeks to evaluate two chemical demulsifiers (diethylene glycol and decylamine) on crude oil sample from the Niger Delta to ascertain the most effective in water separation. Demulsifier concentration used were $80 \%$ and $20 \%$ water. Water-in- oil emulsion was formulated in the ratio of $360 \mathrm{ml}: 40 \mathrm{ml}$ of crude oil and distilled water respectively in the laboratory. The demulsification process was carried out at a temperature of $158 \mathrm{O}$ F. Demulsifier doses of $0.5,1,1.5$, and $2 \mathrm{mls}$ were carried out at centrifugal speed of 300rpm, $600 \mathrm{rpm}, 900 \mathrm{rpm}$ and separation time $(10,20,40,60,100,150 \mathrm{sec})$. Volumes of water separated were recorded and water separation efficiencies were calculated. Results have shown that faster rate of water separation and performance efficiency can be achieved when diethylene glycol is used as demulsifier on crude oil from the Niger Delta region of Nigeria.
\end{abstract}

Keywords: Demulsifiers, emulsion, crude oil

\section{Introduction}

Production of oil is associated with water in oil reservoirs. During flow of oil from the reservoir through the tubing to the well head, emulsion is formed due to turbulent flow of oil and water. Emulsion consist of three types namely: (i) water- in- oil (w/o), water dispersing into the oil which is the continuous phase. (ii) oil-in-water (o/w), oil dispersing into the water which is the continuous phase. (iii) complex emulsions which is the combination of oil in water (o/w) and water in oil (w/ o). The pre-requisites for stable emulsion to form include (i) Two immiscible liquids (ii) Emulsifying agent (iii) Agitation (Schuber and Armbruster, 1992). Emulsions are formed due to Asphaltene, resins, waxes otherwise known as emulsifying agents present in crude oil (Lee, 1999). Chemical demulsifiers used in the treatment of emulsions in the oil and gas industry are either polar (hydrophilic) or non-polar (hydrophobic) (Abdulkadir, 2006). Demulsifier molecules having equal partitioning between aqueous and oil phase are the most efficient demulsifiers (Al-Sabagh et al., 2011). Application of demulsifying agent for emulsion treatment depends on the properties of the crude oil which differ from one another (Hanapi, 2006). During demulsification process, temperature changes cause remarkable changes in the stability of emulsion; emulsions may be inverted or broken (Al-Sabagh et al., 2014). The aim of the study is to determine which of the demulsifiers (diethylene glycol and decylamine) generate better water separation efficiency. 


\section{Materials and Methods}

\subsection{Materials}

The materials used are presented in Table 1 below

\begin{tabular}{|c|c|c|c|}
\hline S/ N & Material Name & S/ N & Material Name \\
\hline 1 & Clock timer & 10 & Diethylene glycol (demulsifier) \\
\hline 2 & Water bath & 11 & Flat bottom flask \\
\hline 3 & Centrifuge & 12 & Decylamine (demulsifier) \\
\hline 4 & Thermometer & 13 & Hydrometer cylinder \\
\hline 5 & Beakers (100 ml) & 14 & Xylene (solvent) \\
\hline 6 & Funnel & 15 & Mixer \\
\hline 7 & Syringe & 16 & Pipette \\
\hline 8 & Centrifuge tube & 17 & Crude oil (from the Niger Delta) \\
\hline 9 & Hydrometer & 18 & Distilled water \\
\hline
\end{tabular}

Table 1: Laboratory Equipment and Material

Properties of chemical demulsifiers used are shown in Table 2 below.

\begin{tabular}{|c|c|c|}
\hline Properties & Diethylene Glycol & Decylamine \\
\hline Molecular formula & $\mathrm{C}_{4} \mathrm{H}_{10} \mathrm{O}_{3}$ & $\mathrm{C}_{10} \mathrm{H}_{23} \mathrm{~N}$ \\
\hline Physical Properties & Colorless liquid & Colorless liquid \\
\hline Density & $1.118 \mathrm{~g} / \mathrm{cm}$ & $0.73 \mathrm{~g} / \mathrm{cm}$ \\
\hline Melting point & $-10.45^{\circ} \mathrm{C}$ & $17^{\circ} \mathrm{C}$ \\
\hline Boiling point & $244-245^{\circ} \mathrm{C}$ & $220.5^{\circ} \mathrm{C}$ \\
\hline
\end{tabular}

Table 2: Properties of Demulsifiers (Diethylene Glycol and Decylamine)

Properties of crude oil samples used from the Niger Delta fields are as shown in Table 3 below

\begin{tabular}{|c|c|}
\hline Crude Oil Properties & Values \\
\hline Colour & Brown \\
\hline Specific gravity, S.G & 0.886 \\
\hline API gravity & $28^{\circ}$ \\
\hline
\end{tabular}

Table 3: Showing Properties of Crude Oil used

\subsection{Methods}

Emulsion preparation. Water-in-oil emulsion (w/o) was prepared in the laboratory at room temperature by mixing crude oil sample and distilled water in the ratio of $90 \%: 10 \%$ or $360 \mathrm{ml}: 40 \mathrm{ml}$ respectively. Hamilton beach electric mixer was used at a speed of $1000 \mathrm{rpm}$ for one hour to obtain a stable emulsion.

Preparation of Demulsifier. Eighty percent (80\%) solution of the chemical demulsifiers (diethylene glycol and decylamine) was prepared as follows:

- Xylene was added into $50 \mathrm{ml}$ graduated flat bottom flask up to $10 \mathrm{ml} \mathrm{level.}$

$40 \mathrm{ml}$ of demulsifier was added to the $50 \mathrm{ml}$ graduated cylinder to make up the total volume.

- The mixture was vigorously agitated with the flask closed and labelled.

- $\quad$ Steps1,2, and 3 were repeated for the other demulsifier (decylamine).

Summary of Mixture

Xylene volume used $=10 \mathrm{ml}$.

Concentrated demulsifier volume used $=40 \mathrm{ml}$.

Total volume of solution $=(40+10)=50 \mathrm{ml}$.

Percentage of xylene in solution $=10 / 50 * 100=20 \%$.

Percentage of demulsifier in solution $=40 / 50 * 100=80 \%$

\subsubsection{Analytical Procedure}

- Emulsion samples in centrifuge tube were placed into centrifuge at a temperature of $1580 \mathrm{~F}$ and set to spin at a given time.

- The volume of water separated is measured in time 10,20,40,60,100, and 150 sec.

- Steps a-b are repeated at various speeds of the centrifuge $300 \mathrm{rpm}, 600 \mathrm{rpm}$ and $900 \mathrm{rpm}$ for demulsifier doses of $0.5 \mathrm{ml}, 1.0 \mathrm{ml}, 1.5 \mathrm{ml}$ and $2.0 \mathrm{ml}$ respectively.

- The above procedures were performed on the two chemical demulsifiers.

- Calculation of water separation efficiency is as follows: \% Water separated (e) =Volume of water separated $\mathrm{ml} /$ Original volume of water in emulsion $\mathrm{ml} * 1003$. Results and Discussion 


\section{Results}

The results of the test performed are shown in the tables below. Tables 3.1 to 3.12 shows volume of water separated from 10 sec.to $150 \mathrm{sec}$, while tables 3.13 to 3.24 shows water separation efficiency of diethylene glycol and decylamine for the same range of time.

\begin{tabular}{|c|c|c|c|c|c|c|}
\hline \multirow{2}{*}{ Demulsifier } & \multicolumn{6}{|c|}{ Time (s) } \\
\cline { 2 - 6 } & $\mathbf{1 0}$ & $\mathbf{2 0}$ & $\mathbf{4 0}$ & $\mathbf{6 0}$ & $\mathbf{1 0 0}$ & $\mathbf{1 5 0}$ \\
\hline Decylamine separated water (ml) & 0.1 & 0.1 & 0.1 & 0.1 & 0.1 & 0.1 \\
\hline $\begin{array}{c}\text { Diethylene glycol (DEG) separated } \\
\text { water (ml) }\end{array}$ & 0.3 & 0.3 & 0.3 & 0.3 & 0.3 & 0.3 \\
\hline
\end{tabular}

Table 4: Demulsification Process Result of 300 Rpm for 0.5 Ml Demulsifier Dose

\begin{tabular}{|c|c|c|c|c|c|c|}
\hline \multirow{2}{*}{ Demulsifier } & \multicolumn{6}{|c|}{ Time (s) } \\
\cline { 2 - 6 } & $\mathbf{1 0}$ & $\mathbf{2 0}$ & $\mathbf{4 0}$ & $\mathbf{6 0}$ & $\mathbf{1 0 0}$ & $\mathbf{1 5 0}$ \\
\hline Decylamine separated water (ml) & 0.15 & 0.15 & 0.15 & 0.15 & 0.15 & 0.15 \\
\hline $\begin{array}{c}\text { Diethylene glycol (DEG) separated } \\
\text { water (ml) }\end{array}$ & 0.35 & 0.35 & 0.35 & 0.35 & 0.35 & 0.35 \\
\hline
\end{tabular}

Table 5: Demulsification Process Result of 300 Rpm for 1.0 Ml Demulsifier Dose

\begin{tabular}{|c|c|c|c|c|c|c|}
\hline \multirow{2}{*}{ Demulsifier } & \multicolumn{7}{|c|}{ Time (s) } \\
\cline { 2 - 7 } & $\mathbf{1 0}$ & $\mathbf{2 0}$ & $\mathbf{4 0}$ & $\mathbf{6 0}$ & $\mathbf{1 0 0}$ & $\mathbf{1 5 0}$ \\
\hline Decylamine separated water (ml) & 0.1 & 0.1 & 0.1 & 0.1 & 0.1 & 0.1 \\
\hline $\begin{array}{c}\text { Diethylene glycol (DEG) separated } \\
\text { water (ml) }\end{array}$ & 0.3 & 0.3 & 0.5 & 1.0 & 1.5 & 2.0 \\
\hline
\end{tabular}

Table 6: Demulsification Process Result of 300 Rpm for 1.5 Ml Demulsifier Dose

\begin{tabular}{|c|c|c|c|c|c|c|}
\hline \multirow{2}{*}{ Demulsifier } & \multicolumn{6}{|c|}{ Time (s) } \\
\cline { 2 - 6 } & $\mathbf{1 0}$ & $\mathbf{2 0}$ & $\mathbf{4 0}$ & $\mathbf{6 0}$ & $\mathbf{1 0 0}$ & $\mathbf{1 5 0}$ \\
\hline Decylamine separated water (ml) & 0.05 & 0.05 & 0.15 & 0.20 & 0.20 & 0.20 \\
\hline $\begin{array}{c}\text { Diethylene glycol (DEG) separated } \\
\text { water (ml) }\end{array}$ & 0.1 & 0.15 & 0.25 & 0.5 & 1.0 & 1.5 \\
\hline
\end{tabular}

Table 7: Demulsification Process Result of 300 Rpm for 2.0 Ml Demulsifier Dose

\begin{tabular}{|c|c|c|c|c|c|c|}
\hline \multirow{2}{*}{ Demulsifier } & \multicolumn{6}{|c|}{ Time (s) } \\
\cline { 2 - 7 } & $\mathbf{1 0}$ & $\mathbf{2 0}$ & $\mathbf{4 0}$ & $\mathbf{6 0}$ & $\mathbf{1 0 0}$ & $\mathbf{1 5 0}$ \\
\hline Decylamine separated water (ml) & 0.1 & 0.1 & 0.1 & 0.1 & 0.1 & 0.1 \\
\hline $\begin{array}{c}\text { Diethylene glycol (DEG) separated } \\
\text { water (ml) }\end{array}$ & 0.3 & 0.3 & 0.3 & 0.3 & 0.3 & 0.3 \\
\hline
\end{tabular}

Table 8; Demulsification Process Result of 600 Rpm for 0.5 Ml Demulsifier Dose

\begin{tabular}{|c|c|c|c|c|c|c|}
\hline \multirow{2}{*}{ Demulsifier } & \multicolumn{6}{|c|}{ Time (s) } \\
\cline { 2 - 6 } & $\mathbf{1 0}$ & $\mathbf{2 0}$ & $\mathbf{4 0}$ & $\mathbf{6 0}$ & $\mathbf{1 0 0}$ & $\mathbf{1 5 0}$ \\
\hline Decylamine separated water (ml) & 0.15 & 0.15 & 0.15 & 0.15 & 0.15 & 0.15 \\
\hline $\begin{array}{c}\text { Diethylene glycol (DEG) separated } \\
\text { water (ml) }\end{array}$ & 0.35 & 0.35 & 0.35 & 0.35 & 0.35 & 0.35 \\
\hline
\end{tabular}

Table 9: Demulsification Process Result of 600 Rpm for 1.0 Ml Demulsifier Dose

\begin{tabular}{|c|c|c|c|c|c|c|}
\hline \multirow{2}{*}{ Demulsifier } & \multicolumn{6}{|c|}{ Time (s) } \\
\cline { 2 - 6 } & $\mathbf{1 0}$ & $\mathbf{2 0}$ & $\mathbf{4 0}$ & $\mathbf{6 0}$ & $\mathbf{1 0 0}$ & $\mathbf{1 5 0}$ \\
\hline Decylamine separated water (ml) & 0.0 & 0.05 & 0.05 & 0.05 & 0.05 & 0.05 \\
\hline $\begin{array}{c}\text { Diethylene glycol (DEG) separated } \\
\text { water (ml) }\end{array}$ & 0.0 & 0.05 & 0.1 & 0.5 & 1.4 & 3.0 \\
\hline
\end{tabular}

Table 10: Demulsification Process Result of 600 Rpm for 1.5 Ml Demulsifier Dose

\begin{tabular}{|c|c|c|c|c|c|c|}
\hline \multirow{2}{*}{ Demulsifier } & \multicolumn{5}{|c|}{ Time (s) } \\
\cline { 2 - 6 } & 10 & 20 & 40 & 60 & 100 & 150 \\
\hline Decylamine separated water (ml) & 0.05 & 0.10 & 0.10 & 0.15 & 0.20 & 0.20 \\
\hline $\begin{array}{c}\text { Diethylene glycol (DEG) separated } \\
\text { water (ml) }\end{array}$ & 0.10 & 0.10 & 0.50 & 1.0 & 1.5 & 1.5 \\
\hline
\end{tabular}

Table 11: Demulsification Process Result of 600 Rpm for 2.0 Ml Demulsifier Dose 


\begin{tabular}{|c|c|c|c|c|c|c|}
\hline \multirow{2}{*}{ Demulsifier } & \multicolumn{7}{|c|}{ Time (s) } \\
\cline { 2 - 6 } & 10 & 20 & 40 & 60 & 100 & 150 \\
\hline Decylamine separated water (ml) & 0.1 & 0.1 & 0.1 & 0.1 & 0.1 & 0.1 \\
\hline $\begin{array}{c}\text { Diethylene glycol (DEG) separated } \\
\text { water (ml) }\end{array}$ & 0.3 & 0.3 & 0.3 & 0.3 & 0.3 & 0.3 \\
\hline
\end{tabular}

Table 12: Demulsification Process Result of 900 Rpm for 0.5 M Demulsifier Dose

\begin{tabular}{|c|c|c|c|c|c|c|}
\hline \multirow{2}{*}{ Demulsifier } & \multicolumn{6}{|c|}{ Time (s) } \\
\cline { 2 - 6 } & 10 & 20 & 40 & 60 & 100 & 150 \\
\hline Decylamine separated water (ml) & 0.15 & 0.15 & 0.15 & 0.15 & 0.15 & 0.15 \\
\hline $\begin{array}{c}\text { Diethylene glycol (DEG) separated } \\
\text { water (ml) }\end{array}$ & 0.35 & 0.35 & 0.35 & 0.35 & 0.35 & 0.35 \\
\hline
\end{tabular}

Table 13: Demulsification Process Result of 900 Rpm for 1.0 Ml Demulsifier Dose

\begin{tabular}{|c|c|c|c|c|c|c|}
\hline \multirow{2}{*}{ Demulsifier } & \multicolumn{6}{|c|}{ Time (s) } \\
\cline { 2 - 6 } & $\mathbf{1 0}$ & $\mathbf{2 0}$ & $\mathbf{4 0}$ & $\mathbf{6 0}$ & $\mathbf{1 0 0}$ & $\mathbf{1 5 0}$ \\
\hline Decylamine separated water (ml) & 0.05 & 0.08 & 0.10 & 0.15 & 0.20 & 0.20 \\
\hline $\begin{array}{c}\text { Diethylene glycol (DEG) separated } \\
\text { water (ml) }\end{array}$ & 0.1 & 0.20 & 0.35 & 0.5 & 1.5 & 2.5 \\
\end{tabular}

Table 14: Demulsification Process Result of 900 Rpm for 1.5 Ml Demulsifier Dose

\begin{tabular}{|c|c|c|c|c|c|c|}
\hline \multirow{2}{*}{ Demulsifier } & \multicolumn{6}{|c|}{ Time (s) } \\
\cline { 2 - 6 } & $\mathbf{1 0}$ & $\mathbf{2 0}$ & $\mathbf{4 0}$ & $\mathbf{6 0}$ & $\mathbf{1 0 0}$ & $\mathbf{1 5 0}$ \\
\hline Decylamine separated water (ml) & 0.05 & 0.10 & 0.15 & 0.20 & 0.20 & 0.20 \\
\hline $\begin{array}{c}\text { Diethylene glycol (DEG) separated } \\
\text { water (ml) }\end{array}$ & 0.5 & 1.0 & 1.5 & 1.5 & 1.5 & 1.5 \\
\hline
\end{tabular}

Table 15: Demulsification Process Result of 900 Rpm for 2.0 Ml Demulsifier Dose

\begin{tabular}{|c|c|c|c|c|c|c|}
\hline Time & $\mathbf{1 0}$ & $\mathbf{2 0}$ & $\mathbf{4 0}$ & $\mathbf{6 0}$ & $\mathbf{1 0 0}$ & $\mathbf{1 5 0}$ \\
\hline Decylamine (e) & 0.25 & 0.25 & 0.25 & 0.25 & 0.25 & 0.25 \\
\hline Diethylene glycol (DEG) (e) & 0.75 & 0.75 & 0.75 & 0.75 & 0.75 & 0.75 \\
\hline
\end{tabular}

Table 16: Water Separation Efficiency Result of 300 Rpm for 0.5 Ml Demulsifier Dose

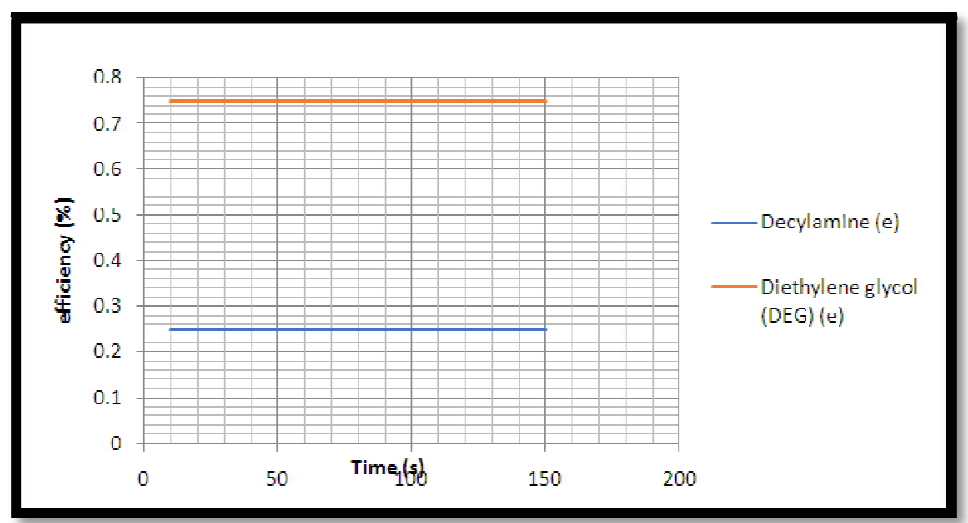

Figure 1: Plot of Water Separation Efficiency for 300rpm in 0.5 M Demulsifier Dose

\begin{tabular}{|c|c|c|c|c|c|c|}
\hline Time & $\mathbf{1 0}$ & $\mathbf{2 0}$ & $\mathbf{4 0}$ & $\mathbf{6 0}$ & $\mathbf{1 0 0}$ & $\mathbf{1 5 0}$ \\
\hline Decylamine (e) & 0.375 & 0.375 & 0.375 & 0.375 & 0.375 & 0.375 \\
\hline Diethylene glycol (DEG) (e) & 0.875 & 0.875 & 0.875 & 0.875 & 0.875 & 0.875 \\
\hline
\end{tabular}

Table 17: Water Separation Efficiency Result of 300 Rpm for 1.0 Ml Demulsifier Dose 


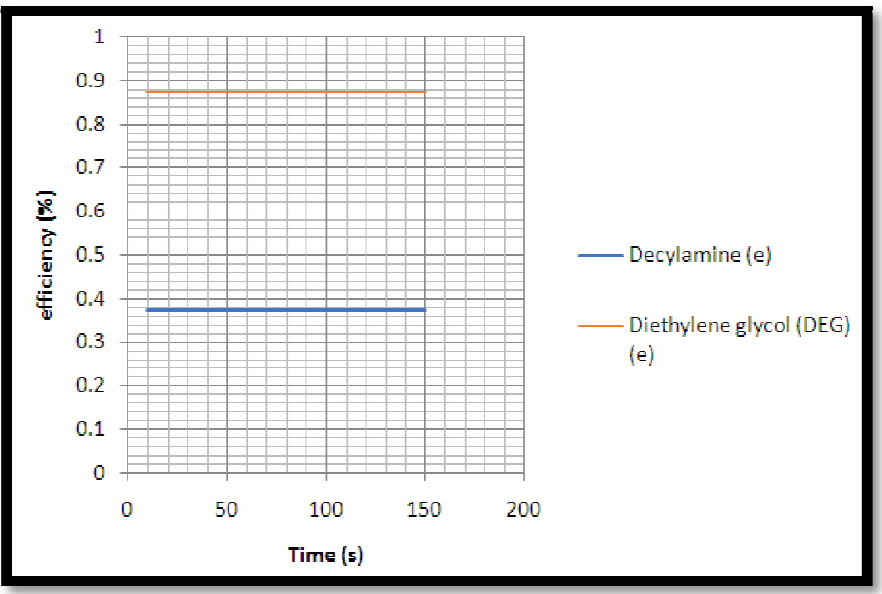

Figure 2: Plot of Water Separation Efficiency for 300rpm in $1.0 \mathrm{M}$ Demulsifier Dose

\begin{tabular}{|c|c|c|c|c|c|c|}
\hline Time & $\mathbf{1 0}$ & $\mathbf{2 0}$ & $\mathbf{4 0}$ & $\mathbf{6 0}$ & $\mathbf{1 0 0}$ & $\mathbf{1 5 0}$ \\
\hline Decylamine (e) & 0.25 & 0.25 & 0.25 & 0.25 & 0.25 & 0.25 \\
\hline Diethylene glycol (DEG) (e) & 0.75 & 0.75 & 1.25 & 2.5 & 3.75 & 5 \\
\hline
\end{tabular}

Table 18: Water Separation Efficiency Result of 300 Rpm for 1.5 Ml Demulsifier Dose

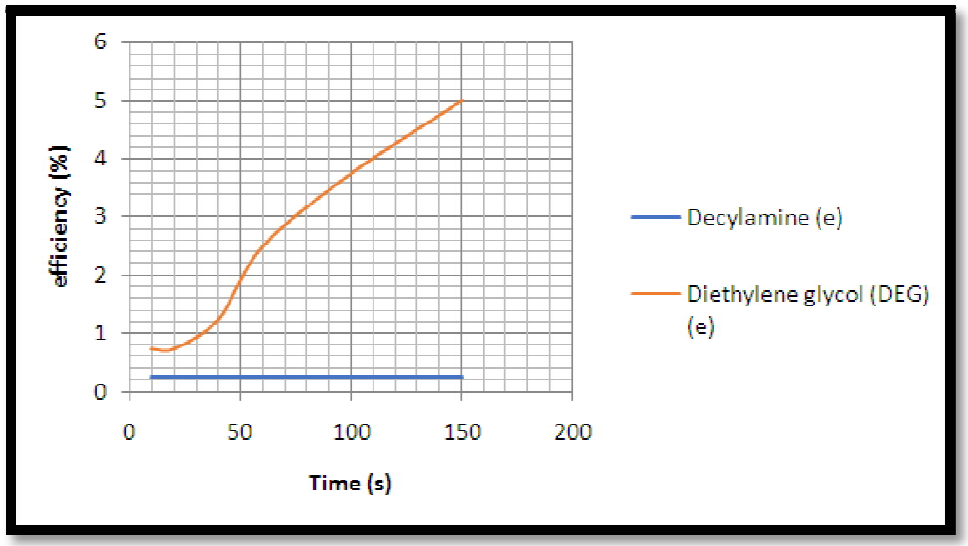

Figure 3: Plot of Water Separation Efficiency for 300rpm in 1.5 Ml Demulsifier Dose

\begin{tabular}{|c|c|c|c|c|c|c|}
\hline Time & $\mathbf{1 0}$ & $\mathbf{2 0}$ & $\mathbf{4 0}$ & $\mathbf{6 0}$ & $\mathbf{1 0 0}$ & $\mathbf{1 5 0}$ \\
\hline Decylamine (e) & 0.125 & 0.125 & 0.375 & 0.5 & 0.5 & 0.5 \\
\hline Diethylene glycol (DEG) (e) & 0.25 & 0.375 & 0.625 & 1.25 & 2.5 & 3.75 \\
\hline
\end{tabular}

Table 19: Water Separation Efficiency Result of 300

Rpm for 2.0 Ml Demulsifier Dose

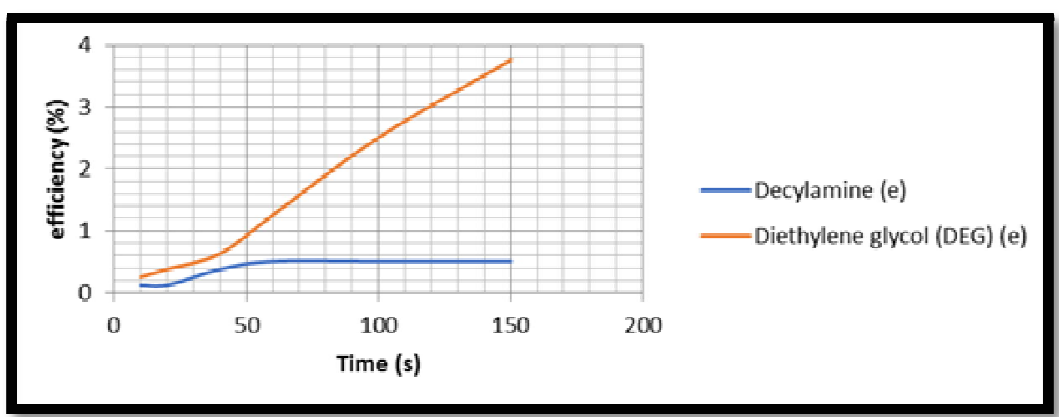

Figure 4: Plot of Water Separation Efficiency for 300rpm in 2.0 Ml Demulsifier Dose

\begin{tabular}{|c|c|c|c|c|c|c|}
\hline Time & $\mathbf{1 0}$ & $\mathbf{2 0}$ & $\mathbf{4 0}$ & $\mathbf{6 0}$ & $\mathbf{1 0 0}$ & $\mathbf{1 5 0}$ \\
\hline Decylamine (e) & 0.25 & 0.25 & 0.25 & 0.25 & 0.25 & 0.25 \\
\hline Diethylene glycol (DEG) (e) & 0.75 & 0.75 & 0.75 & 0.75 & 0.75 & 0.75 \\
\hline
\end{tabular}

Table 20: Water Separation Efficiency Result of $600 \mathrm{Rpm}$ for 0.5ml Demulsifier Dose 


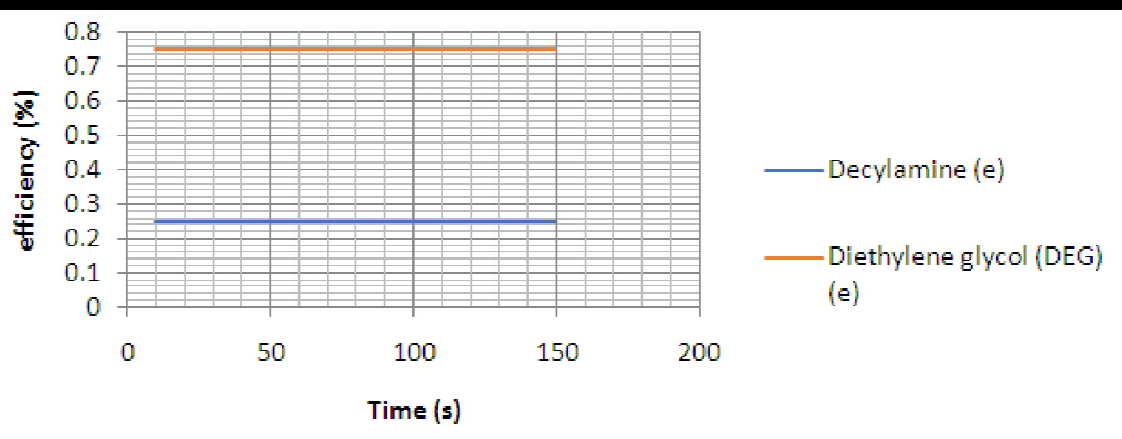

Figure 5: Plot of Water Separation Efficiency for 600rpm in 0.5 M Demulsifier Dose

\begin{tabular}{|c|c|c|c|c|c|c|}
\hline Time & $\mathbf{1 0}$ & $\mathbf{2 0}$ & $\mathbf{4 0}$ & $\mathbf{6 0}$ & $\mathbf{1 0 0}$ & $\mathbf{1 5 0}$ \\
\hline Decylamine (e) & 0.375 & 0.375 & 0.375 & 0.375 & 0.375 & 0.375 \\
\hline Diethylene glycol (DEG) (e) & 0.875 & 0.875 & 0.875 & 0.875 & 0.875 & 0.875 \\
\hline
\end{tabular}

Table 21: Water Separation Efficiency Result of 600 Rpm for 1.0 Ml Demulsifier Dose

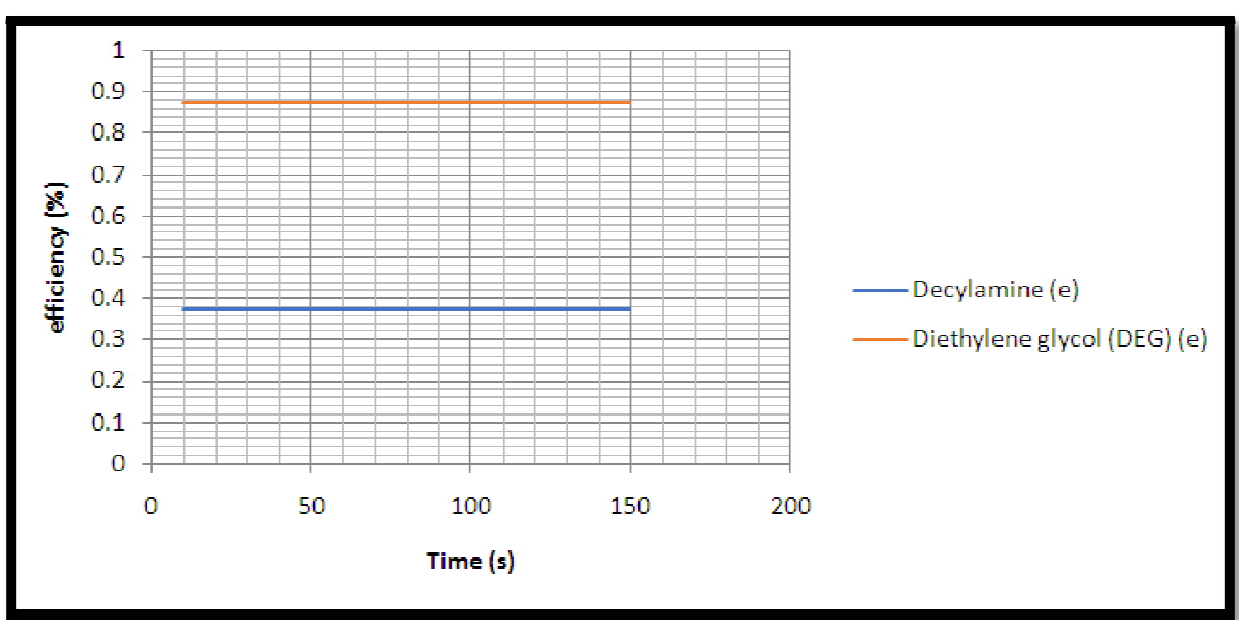

Figure 6: Plot of Water Separation Efficiency for 600rpm In 1.0 Ml Demulsifier Dose

\begin{tabular}{|c|c|c|c|c|c|c|}
\hline Time & $\mathbf{1 0}$ & $\mathbf{2 0}$ & $\mathbf{4 0}$ & $\mathbf{6 0}$ & $\mathbf{1 0 0}$ & $\mathbf{1 5 0}$ \\
\hline Decylamine (e) & 0 & 0.125 & 0.125 & 0.125 & 0.125 & 0.125 \\
\hline Diethylene glycol (DEG) (e) & 0 & 0.125 & 0.25 & 1.25 & 3.5 & 7.5 \\
\hline
\end{tabular}

Table 22: Water Separation Efficiency Result of 600 Rpm for 1.5 Ml Demulsifier Dose

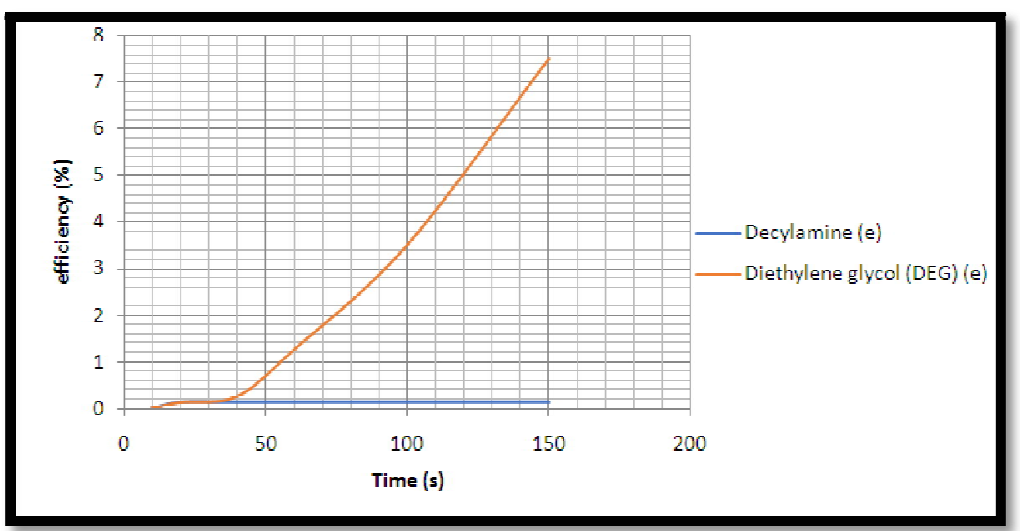

Figure 7: Plot of Water Separation Efficiency for 600rpm in 1.5 M Demulsifier Dose

\begin{tabular}{|c|c|c|c|c|c|c|}
\hline Time & $\mathbf{1 0}$ & $\mathbf{2 0}$ & $\mathbf{4 0}$ & $\mathbf{6 0}$ & $\mathbf{1 0 0}$ & $\mathbf{1 5 0}$ \\
\hline Decylamine (e) & 0.125 & 0.25 & 0.25 & 0.375 & 0.5 & 0.5 \\
\hline Diethylene glycol (DEG) (e) & 0.25 & 0.25 & 1.25 & 2.5 & 3.75 & 3.75 \\
\hline
\end{tabular}

Table 23: Water Separation Efficiency Result of 600 Rpm for 2.0 Ml Demulsifier Dose 


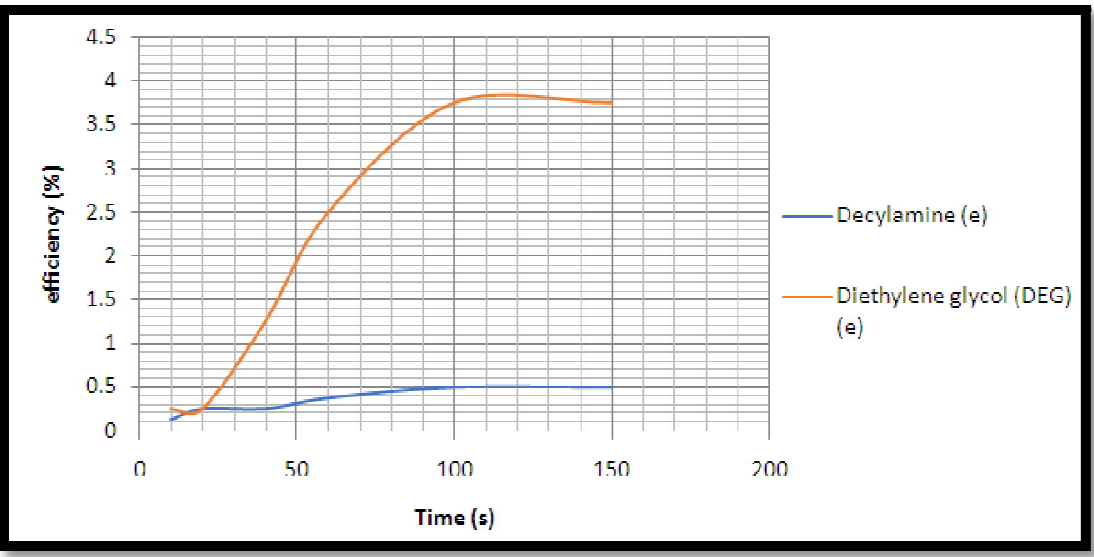

Figure 8: Plot of Water Separation Efficiency for 600rpm in 2.0 Ml Demulsifier Dose

\begin{tabular}{|c|c|c|c|c|c|c|}
\hline Time & $\mathbf{1 0}$ & $\mathbf{2 0}$ & $\mathbf{4 0}$ & $\mathbf{6 0}$ & $\mathbf{1 0 0}$ & $\mathbf{1 5 0}$ \\
\hline Decylamine (e) & 0.25 & 0.25 & 0.25 & 0.25 & 0.25 & 0.25 \\
\hline Diethylene glycol (DEG) (e) & 0.75 & 0.75 & 0.75 & 0.75 & 0.75 & 0.75 \\
\hline
\end{tabular}

Table 24: Water Separation Efficiency Result of 900 Rpm for 0.5 Ml Demulsifier Dose

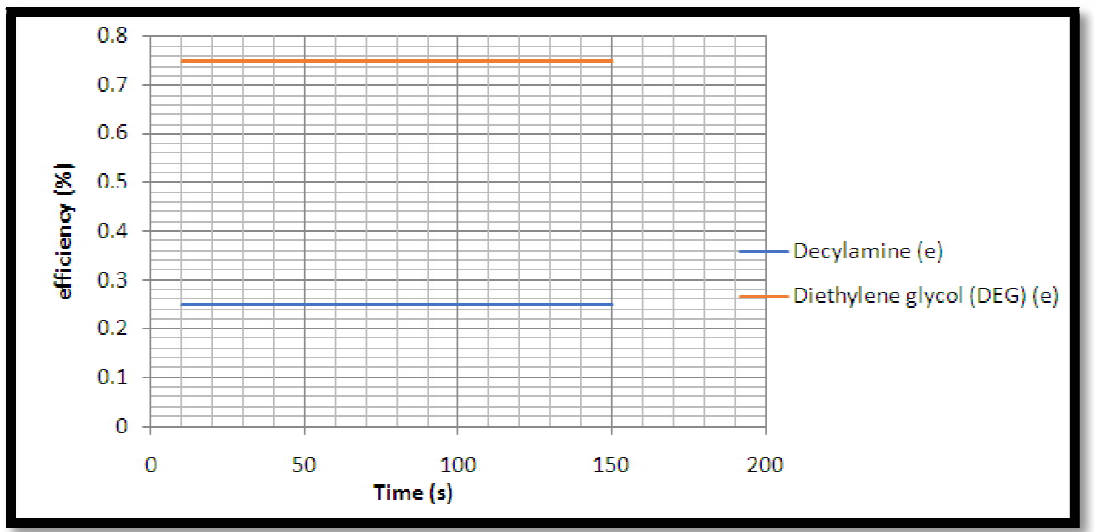

Figure 9: Plot of water separation efficiency for 900rpm in $0.5 \mathrm{ml}$ demulsifier dose

\begin{tabular}{|c|c|c|c|c|c|c|}
\hline Time & $\mathbf{1 0}$ & $\mathbf{2 0}$ & $\mathbf{4 0}$ & $\mathbf{6 0}$ & $\mathbf{1 0 0}$ & $\mathbf{1 5 0}$ \\
\hline Decylamine (e) & 0.375 & 0.375 & 0.375 & 0.375 & 0.375 & 0.375 \\
\hline Diethylene glycol (DEG) (e) & 0.875 & 0.875 & 0.875 & 0.875 & 0.875 & 0.875 \\
\hline
\end{tabular}

Table 25: Water Separation Efficiency Result of 900 Rpm for 1.0 Ml Demulsifier Dose

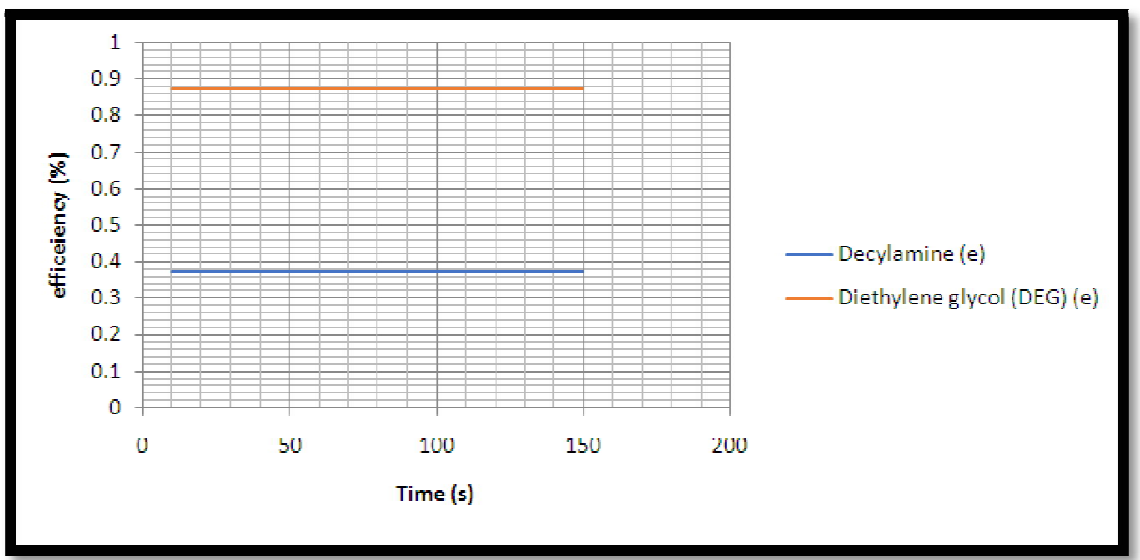

Figure 10: Plot of Water Separation Efficiency for 900rpm in 1.0 M Demulsifier Dose

\begin{tabular}{|c|c|c|c|c|c|c|}
\hline Time & $\mathbf{1 0}$ & $\mathbf{2 0}$ & $\mathbf{4 0}$ & $\mathbf{6 0}$ & $\mathbf{1 0 0}$ & $\mathbf{1 5 0}$ \\
\hline Decylamine (e) & 0.125 & 0.2 & 0.25 & 0.375 & 0.5 & 0.5 \\
\hline Diethylene glycol (DEG) (e) & 0.25 & 0.5 & 0.875 & 1.25 & 3.75 & 6.25 \\
\hline
\end{tabular}

Table 26: Water Separation Efficiency Result of 900 Rpm for 1.5 Ml Demulsifier Dose 


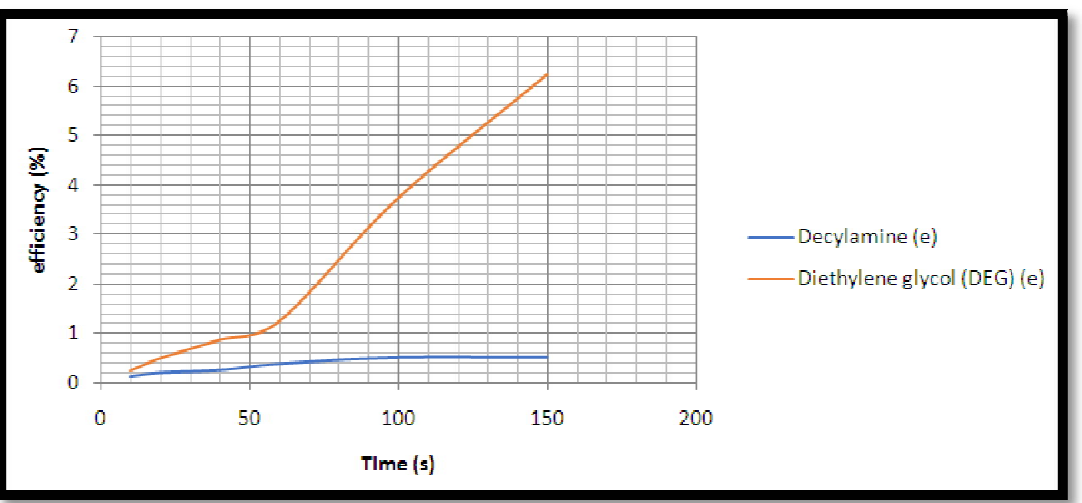

Figure 11: Plot of Water Separation Efficiency for 900rpm in 1.5 Ml Demulsifier Dose

\begin{tabular}{|c|c|c|c|c|c|c|}
\hline Time & $\mathbf{1 0}$ & $\mathbf{2 0}$ & $\mathbf{4 0}$ & $\mathbf{6 0}$ & $\mathbf{1 0 0}$ & $\mathbf{1 5 0}$ \\
\hline Decylamine (e) & 0.125 & 0.25 & 0.375 & 0.5 & 0.5 & 0.5 \\
\hline Diethylene glycol (DEG) (e) & 1.25 & 2.5 & 3.75 & 3.75 & 3.75 & 3.75 \\
\hline
\end{tabular}

Table 27: Water Separation Efficiency Result of 900 Rpm for 2.0 Ml Demulsifier Dose

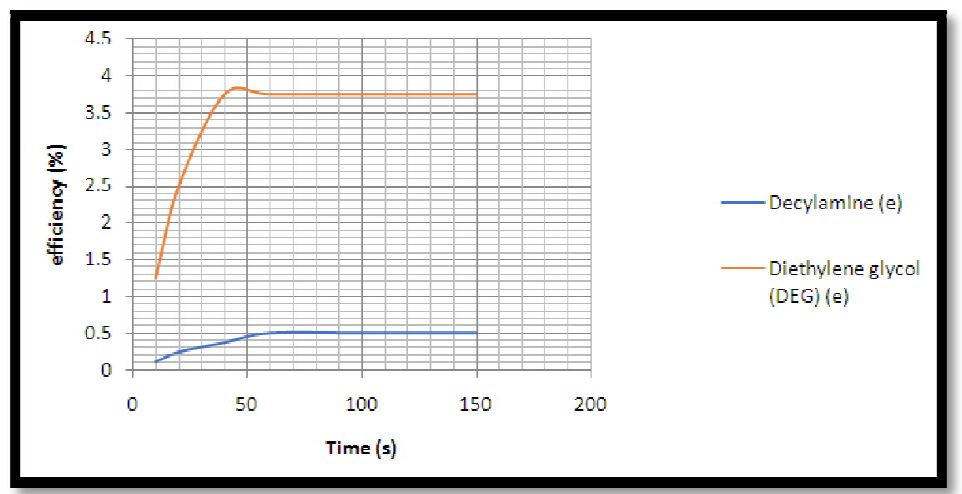

Figure 12: Plot of Water Separation Efficiency for 900rpm in 2.0 M Demulsifier Dose

\section{Discussion of Results}

In Table 18 at centrifuge speed of 300rpm for $1.5 \mathrm{ml}$ demulsifier dose, water separation efficiency was $5 \mathrm{ml}$ in 150sec with diethylene glycol and 0.5 in $150 \mathrm{sec}$ with decylamine while in Table 22 at a speed of $600 \mathrm{rpm}$ for $1.5 \mathrm{ml}$ demulsifier dose, water separation efficiency was $7.5 \mathrm{ml}$ in $150 \mathrm{sec}$ with diethylene glycol and $0.125 \mathrm{ml}$ in $150 \mathrm{sec}$ with decylamine. In Table 26, at centrifuge speed of 900 rpm for $1.5 \mathrm{ml}$ demulsifier dose, water separation efficiency was $6.25 \mathrm{ml}$ in 150sec with diethylene glycol and $0.5 \mathrm{ml}$ with decylamine.

\section{Conclusion}

Rate of water separation is quicker and efficiency in terms of performance can be realized when diethylene glycol is used as demulsifier on crude oil sample from the Niger Delta region of Nigeria.

\section{References}

i. Abdulkadir, M. (2006). Comparative Analysis of the Effect of Demulsifiers in the Treatment of Crude Oil Emulsion. ARPN J. Eng. Appl. Sci. 5:6.

ii. AI-Sabagh, A.M, Kandile, N.G, EI-Ghazawy, R.A, Noor EI-Din, M.R. (2011). Synthesis and evaluation of some new demulsifiers based on bisphenols for treating water-in-crude oil emulsions. EJP (2011) 20, 67-77

iii. AI-Sabagh, A.M., Nasser, N.M., Khamis, E.A. (2014). Demulsification of crude oil emulsions using ethoxylated aliphatic amine polyesters as novel demulsifiers. IJSR, Volume 3 Issue 12, December 2014.

iv. Hanapi BM (2006). Study on Demulsifier formulation for treating Malaysian Crude Oil Emulsion. MSc. Thesis, University Technology

v. Lee, R. F. (1999). Agents Which Promote and Stabilize Water-In-Oil Emulsions. Spill Science \& Technology Bulletin. Elsevier Science Ltd. 5 (2): pg117-12

vi. Schubert, Hand Armbruster, H (1992). Principles of formation and stability of emulsions 